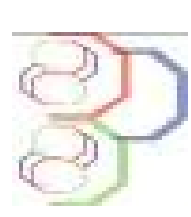

Journal of Applied Biosciences 127: 12794-12803

ISSN 1997-5902

\title{
Influence du moment d'application du NPK sur la croissance et le rendement du maïs (Zea mays L.) installé sur un ferralsol
}

\author{
Ilunga Tabu Hugues ${ }^{*}$, Banza Mukalay John1, Lukusa Mukoka Lucky², Mukunto Kimonge Ismael ${ }^{3}$, \\ Malonga Hugor L'kisaten ${ }^{4}$, Kanyenga Lububo Antoine ${ }^{1,5}$, Nyembo Kimuni Luciens ${ }^{1}$ \\ ${ }^{1}$ Faculté des Sciences Agronomiques, Université de Lubumbashi, Lubumbashi, RD Congo ; \\ 2Faculté des Sciences Agronomiques, Université de Kolwezi, Kolwezi, RD Congo ; \\ ${ }^{3}$ Département des sciences agrovétérinaires, Institut Pédagogique de Lubumbashi, Lubumbashi, RD Congo ; \\ ${ }^{4}$ Institut Supérieur de Techniques appliquées de Lubumbashi, Lubumbashi, RD Congo ; \\ ${ }^{5}$ Centre International pour l'agriculture Tropicale (CIAT-HarvestPlus), Kalambo, Bukavu, RD Congo \\ *Auteur Correspondant : ilungahugues8@gmail.com; Tabul@unilu.ac.cd
}

Original submitted in on $2^{\text {nd }}$ April 2018. Published online at www.m.elewa.org on $31^{\text {st }}$ July 2018 https://dx.doi.org/10.4314/jab.v127i1.4

\section{RÉSUMÉ}

Objectif : La province du Haut Katanga est dominée par des sols ferralitiques, une des sources majeures des faibles rendements du maïs dans cette contrée. Pour pallier aux problèmes d'infertilités de ces sols et accroitre la productivité, les producteurs recourent essentiellement aux engrais minéraux (NPK). Ces derniers sont généralement appliqués au moment de semis. Cependant, avec les perturbations climatiques actuelles, le moment de leur application devient une préoccupation. Ainsi, ce travail a été initié en vue d'évaluer les effets de différents moments d'apport d'engrais ternaire NPK sur le comportement de deux génotypes de maïs (Unilu et LY 1001-10).

Méthodologie and résultats : L'essai a été installé suivant un dispositif en split plot comprenant d'une part quatre moments $(0,10,20$ et 30 jours après levée) d'application du NPK et un témoin (sans engrais) et d'autre part deux génotypes (Unilu et LY 1001-10) répétés 4 fois. Les résultats obtenus ont montré que l'application d'engrais de fond (NPK) permet d'obtenir des plantes de grandes tailles, ayant un poids de l'épi égrené ainsi que le rendement élevé. Cependant, l'application du NPK au moment de semis induit un faible taux de levée, un grand poids de l'épi mais similaire au NPK appliqué dix jours après levée. Toutefois, le poids de l'épi diminue au fur et à mesure que l'application de l'engrais avoisine le jour à la floraison et la perte devient de plus en plus grande.

Conclusion et application des résultats : La perte du poids de l'épi égrené est plus élevée, dans la province du Haut-Katanga, lorsque le maïs est cultivé sans engrais et qu'il est préférable de cultivé sans engrais que d'apporter l'engrais vers le 30ème jour après levée. L'application du NPK au moment de semis est très avantageuse si elle n'est pas suivie d'une période sèche. Dans le cas contraire, apporter le NPK 10 jours après levée est une technique à encourager et que l'allongement du décalage d'application est néfaste pour la production.

Mots clés : NPK, maïs, génotype, levée, ferralsol 
Influence of NPK application moment on growth and yield of maize (Zea mays L.) installed on ferralsol ABSTRACT

Objective : The Haut-Katanga province is dominated by ferralitic soils, a major source of low corn yields. To remedy the infertility problems of these soils and to increase productivity, producers mainly use mineral fertilizers (NPK). Generally, NPK is applied at seedling. However, with the current climate disturbances, the timing of its application becomes a concern. Thus, this work was initiated to evaluate the effects of different ternary NPK fertilization times on the behavior of two maize genotypes (Unilu and LY 1001-10).

Methodology and results: The trial was installed in a split plot device comprising, firstly four moments $(0,10,20$ and 30 days after emergence) of NPK application and a control (without fertilizer) and, on the other hand, two genotypes (Unilu and LY 1001-10) repeated 4 times. The results obtained showed that NPK application allows large plants with a high ginned cob's weight and high yield. However, the application of NPK at seedling induces a low emergence rate, a large weight of ginned cob but similar to NPK applied ten days after emergence. However, the ginned cob's weight decreases as the application of the fertilizer approaches the day of flowering and the loss becomes high.

Conclusion and application of results: The loss of ginned cob's weight is higher in the Haut-Katanga province when the maize is grown without fertilizer and it is better to grow without fertilizer than to bring fertilizer around the 30th day after emergence. The application of NPK at seedling is very advantageous if it is not followed by a dry period. If not, spreading the NPK 10 days after emergence is a technique to promote.

Keywords: NPK, maize, genotype, emergence, ferralsol

\section{INTRODUCTION}

L'analyse de la situation et des perspectives sur la sécurité alimentaire en Afrique sub-saharienne montre un écart croissant entre les besoins de consommation et les disponibilités alimentaires au niveau global des pays, des ménages et des individus. Cette situation entraine une malnutrition et une sous-alimentation marquée (Dembele, 2001). Cette situation pourrait s'empirer d'ici 2050 suite à un accroissement très important de la population dans ladite région (Lutz et al., 2001 ; Ezeh et al., 2012).Augmenter la production d'aliments de base reste l'unique possibilité de lutter contre l'augmentation du taux de pauvreté et de sousalimentation. Or, le maïs (Zea mays L.) est l'une de trois céréales les plus cultivées au monde (Nyembo, 2010). Ces fabuleuses statistiques sont dues au fait que toutes les parties de la plante ont une valeur économique (Nyembo et al., 2014). En République Démocratique du Congo (RDC) en général et dans la province du la province du Haut-Katanga en particulier, la grande production de cette denrée est consommée sous forme de farine et constitue ainsi la nourriture de base préférée par la majorité de la population (Nyembo, 2010). Malgré l'importance accordée à cette culture, sa production n'arrive toujours pas à subvenir aux besoins de cette population devenant ainsi dépendante des importations en provenance de l'Afrique australe (Nyembo et al., 2014). Les faibles rendements obtenus, généralement inférieurs à $1000 \mathrm{~kg}$ par hectare (Nyembo et al., 2015), en sont la cause. Ceci est en partie dû à la faible utilisation de génotypes améliorées et d'intrants agricoles, à l'importance des dommages causés par divers ravageurs et aux mauvaises pratiques agricoles (Nyembo et al., 2014). Selon Useni et al. (2013), la gestion inadéquate de la nutrition des plantes et la faible fertilité du sol sont les principaux facteurs responsables de la baisse des rendements. Or, les sols de la Province du Haut-Katanga en général et ceux de la ville de Lubumbashi en particulier appartiennent à la catégorie des sols ferralitiques caractérisés par un pH acide (Cunha et al., 2009), une faible teneur en matière organique (Kasongo et al., 2013), la présence d'argiles à faible activité principalement la kaolinite et les sesquioxydes (Qafoku et al., 2004) ainsi que l'indisponibilité de nutriments tels que $P$ et $N$ lessivés par l'érosion éolienne et hydrique (Amede, 2003 ; Henao et Baanante, 2006). Par conséquent, ces sols sont de plus en plus improductifs et, dans les cas extrêmes, l'activité agricole n'y est réalisable qu'avec des 
apports d'éléments nutritifs considérables (Useni et al., 2013). Devant ce fait, llunga et al. (2015) et llunga et al. (2016) ont suggéré l'utilisation de la matière organique. Toutefois, la libération des éléments par la matière organique est progressive et lente (Bot et Benites, 2005), parfois la plante n'en bénéficie même pas au moment voulu (Giroux et al., 2007; Pang et Letey, 2000). De plus, leur utilisation requiert de grande quantité, ce qui limite leur usage. C'est ainsi que d'autres études se sont penchés sur l'utilisation des engrais minéraux (Nyembo et al., 2012 ; Nyembo et al., 2013 ; Useni et al., 2012; Useni et al., 2013; Nyembo et al., 2015). Ces derniers ont l'avantage de disponibiliser, pour la plante, les éléments minéraux le plus rapidement, favorisant ainsi une meilleure production et

\section{MATÉRIELS ET MÉTHODES}

Localisation du site : L'étude a été menée à la ferme Kassapa, station de recherche de l'Université de Lubumbashi (altitude de $1256 \mathrm{~m}$, latitude $11^{\circ} 35^{\prime}$ S et une longitude de $27^{\circ} 24^{\prime}$ E) située dans la ville de Lubumbashi, au cours de la saison agricole 2016-2017. Cette ville connait un climat du type CW6 selon la productivité (Nyembo et al., 2012). Dans la maïsiculture Haut-Katangaise, l'urée et le NPK sont des engrais généralement utilisés. Ce dernier est souvent apporté au moment de semis dans les grandes exploitations ou après semis par les paysans. Cette dernière pratique est réconfortée par des perturbations climatiques enregistrées au cours de ces dernières décennies. Mais jusqu'à quel moment après semis peut-on appliquer le NPK comme engrais de fond? Ainsi l'objectif général de ce travail est d'évaluer le comportement du maïs sous l'effet de différents moments d'apport d'engrais tertiaire. Spécifiquement, il s'agira de (i) déterminer la période optimale de ces apports d'engrais ternaires qui rentabilisent la culture et (ii) déterminer les périodes à laquelle l'engrais est inefficace.

classification de Koppen (FAO, 2005a) et est par l'alternance d'une saison de pluie, allant de novembre à mars, et une saison sèche allant de mai à septembre. Juillet et Aout sont les mois les plus secs (Kasongo et al., 2013). Les conditions climatiques ayant prévalu au cours de l'essai sont données dans le tableau 1. Ci-dessous

Tableau 1. Données climatiques de la période d'expérimentation issue de l'Agence nationale de météorologie et de télédétection par satellite/ Station de Luano

\begin{tabular}{l|l|r|r|r|r|r|r}
\hline \multicolumn{1}{l}{\begin{tabular}{l} 
Période climatique et paramètre \\
\multicolumn{1}{l|}{ climatique }
\end{tabular}} & 2016 & \multicolumn{5}{c}{2017} \\
\cline { 3 - 8 } Précipitation & Décembre & Janvier & Février & Mars & Avril & Mai \\
\hline & Nombrité (mm) & 159 & 181,3 & 399,4 & 139,5 & 207,8 & 34,4 \\
& pluvieux & 16 & 18 & 22 & 13 & 8 & 5 \\
Température jours & Maximum (\%) & 26,6 & 25,07 & 24,6 & 24,5 & 24,8 & 25,9 \\
& Moyenne (\%) & 22 & 21,3 & 20,9 & 20,6 & 20,5 & 18,7 \\
& Minimum (\%) & 17,4 & 17,2 & 17,3 & 16,71 & 16,2 & 11,5 \\
\multicolumn{1}{l}{ Humidité relative $(\%)$} & 79 & 88 & 87 & 87 & 85 & 64 \\
\hline
\end{tabular}

La quantité élevée des précipitations a été enregistrée au mois de février. Le mois de décembre a enregistré 16 jours de pluie avec plus d'une semaine sèche. Par contre l'humidité relative et la température les plus élevées ont été enregistrée respectivement au mois de janvier et décembre. Du point de vue édaphique, les sols de la ferme Kasapa et de la ville de Lubumbashi en général appartiennent à la catégorique de ferralsol, acides avec un pH à l'eau oscillant autour de 5,2 (Kasongo et al., 2013). L'analyse, effectuée au laboratoire Pédologique de la Faculté de Sciences Agronomiques de l'Université de Lubumbashi, de l'échantillon composite du sol, a donné les résultats suivants : $\mathrm{pH}$ eau $=5,96 ; \mathrm{N}$ total $=0,08 \% ; \mathrm{P}$ disponible $=7,1716 \% ; \mathrm{K}$ total $=0,4 \% ; \mathrm{Fe}$ total $=1,7 \%$ et $\mathrm{Cu}=171 \mathrm{ppm}$.

Matériel : Le matériel végétal utilisé était composé de deux génotypes de maïs (Unilu et LY 1001-10). Le génotype Unilu, mise au point par Faculté des Sciences Agronomiques de l'Université de Lubumbashi. II s'agit d'un génotype amélioré à pollinisation ouverte de couleur blanche. Elle est plus préférée pour sa résistance aux maladies (helminthosporiose, cercosporiose et striure), ravageurs, son potentiel élevé de rendement ( 7 à 8,5 t.ha-1 à une densité de 53333 plants ha-1) et la faible 
taille des plantes qui les rend moins sensible à la verse (Nyembo et al., 2013). Cependant, le génotype LY 100110, génotype orange en provenance de HarvestPlus, a été choisi pour sa richesse en Provitamine A. Le NPK 17 - 17 - 17 et l'urée (46\% d'azote) ont été utilisés comme fertilisants.

\section{Méthode}

Conduite de l'essai : L'essai a été installé suivant un dispositif en split plot. Les traitements, en quatre répétitions, consistaient en deux génotypes de maïs (Unilu et LY 1001-10) et quatre moments d'apport de NPK qui ont été à un témoin sans engrais minéral. Le NPK a été apporté à $0,10,20$ et 30 jours après levée $(\mathrm{JAL})$ à raison de $6 \mathrm{~g}$ par poquet soit $320 \mathrm{~kg}^{*}$ ha-1. Après labour et hersage, le semis est intervenu en date du $10 / 12 / 2016$, aux écartements de $75 \mathrm{~cm} \times 25 \mathrm{~cm}$, soit une densité de 53333 plants $^{*} \mathrm{ha}^{-1}$. Les soins d'entretien ont consisté aux sarclages et buttage. Au total 3 sarclages

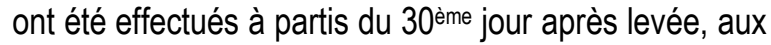
intervalles de 15 jours. L'urée a été apportée au $35^{\text {ème }}$ jour après levée.

Paramètres observés et traitement statistique : Les observations ont portés sur les paramètres végétatifs et les paramètres de rendement. Les paramètres végétatifs observés comprenaient. En cours de l'essai, le taux de levée a été déterminé par le rapport de nombre de

\section{RÉSULTATS}

Effets du moment d'apport d'engrais de fond sur les paramètres végétatifs: L'examen du Tableau 2.cidessous montre que les deux génotypes de maïs (Unilu et LY 1001-10) sont similaires sur tous les paramètres végétatifs étudiés excepté le nombre de jour à la floraison mâle $(P=0,000)$ ainsi que la hauteur de la plante $(P=0,001)$. Le génotype $L Y 1001-10$ a affiché une très faible taille et une floraison tardive que le génotype Unilu. La comparaison du moment d'apport d'engrais montre des différences significatives sur le taux de levée plantes qui ont levé $\times 100$ sur le nombre de graines semées par parcelle. En cours de végétation, la durée semis floraison mâle (durée entre le jour de semis et le jour où au moins $50 \%$ des plantes sont en floraison), la hauteur de la plante ainsi que la hauteur à l'insertion de l'épi ont été mesurés. A la récolte, les épis des maïs ont été récoltés sur les trois lignes du milieu et les paramètres de rendement ont été ajustés au taux d'humidité de $14 \%$. Les observations ont porté sur le poids des grains par épi (poids de grains récoltés sur trois lignes sur le nombre d'épis trouvés sur lesdites lignes), le poids de 1000 grains (grains pris sur la partie médiane de l'épi) et le rendement en maïs grain. Les données brutes sur les paramètres végétatifs et de rendement ont été analysées à l'aide du logiciel Ri386 3.3.2. L'analyse de la variance (ANOVA) a été effectuée afin de déceler les différences entre traitements et la comparaison des moyennes, à l'aide du test de Tukey, a permis de déceler le meilleur traitement. La quantification de la perte due au retard d'apport d'engrais a été obtenue en utilisant la formule PPEE $E_{(t)}=100-R_{(t)}$ où $\operatorname{PPEE}_{(t)}$ est la perte $d u$ poids moyen de l'épi égrené d'un traitement (\%) et $R_{(t)}$ le rapport en pourcentage entre le Poids moyen de l'épi égrené dans les parcelles ayant reçu l'engrais au moment de semis et le Poids moyen de l'épi égrené dans ledit traitement.

$(P=0,000)$ et la hauteur de la plante $(P=0,000)$. Le nombre de plante sur les parcelles fertilisées après semis et les parcelles non fertilisées a été similaire mais inférieur à celui obtenu sur les parcelles fertilisées au moment de semis. Quant à la taille de la plante, elle a été similaire sur toutes les parcelles fertilisées et seules les parcelles sans engrais ont affiché des plantes de faibles tailles. L'analyse des effets combinés montre qu'il n'existe pas d'interaction entre le génotype et le moment d'apport d'engrais pour tous les paramètres étudiés. 


\section{Ilunga et al, J. Appl. Biosci. 2018 Influence du moment d'application du NPK sur la croissance et le rendement}

du maïs (Zea mays L.) installé sur un ferra sol

Tableau 3 : Influence de la variété et du moment d'apport d'engrais de fond sur les paramètres de rendement du maïs. Les moyennes ne partageant pas la même lettre sont sensiblement différentes.

\begin{tabular}{|c|c|c|c|c|c|c|c|}
\hline Variété & Moment & PEE (c & & PMG ( & & Ren & lent $\left(t^{*} h a^{-1}\right)$ \\
\hline & $0 \mathrm{JAL}$ & 128,2 & $\pm 4,9$ & 205,2 & $\pm 12,0$ & & $\pm 0,3$ \\
\hline & $10 \mathrm{JAL}$ & 108,7 & $\pm 13,5$ & 244,6 & $\pm 41,6$ & 4,5 & $\pm 1,4$ \\
\hline & $20 \mathrm{JAL}$ & 103,6 & $\pm 16,3$ & 231,8 & $\pm \quad 35,5$ & 4,2 & $\pm \quad 0,9$ \\
\hline 음 & $30 \mathrm{JAL}$ & 67,4 & $\pm 32,5$ & 197,2 & $\pm \quad 44,2$ & 2,7 & $\pm 1,6$ \\
\hline 호 & Sans Engrais & 55,0 & $\pm \quad 13,9$ & 222,2 & $\pm \quad 13,8$ & 2,3 & $\pm \quad 0,7$ \\
\hline $\bar{\Sigma}$ & Moyenne & 92,6 & $\pm \quad 32,4 a$ & 220,2 & $\pm 33,8 \mathrm{~b}$ & 3,4 & $\pm \quad 1,3$ \\
\hline & $0 \mathrm{JAL}$ & 160,8 & $\pm 12,1$ & 306,1 & $\pm 26,5$ & 3,4 & $\pm \quad 0,9$ \\
\hline & $10 \mathrm{JAL}$ & 129,4 & $\pm 6,3$ & 300,3 & $\pm 26,7$ & 6,6 & $\pm \quad 0,7$ \\
\hline & $20 \mathrm{JAL}$ & 103,6 & $\pm 21,5$ & 305,4 & $\pm 44,1$ & 4,4 & $\pm \quad 1,1$ \\
\hline & $30 \mathrm{JAL}$ & 104,0 & $\pm \quad 38,5$ & 300,6 & $\pm \quad 44,7$ & 4,2 & $\pm \quad 2,3$ \\
\hline 3 & Sans Engrais & 72,6 & $\pm \quad 30,9$ & 310,3 & $\pm \quad 47,2$ & 3,1 & $\pm 1,6$ \\
\hline $\bar{\Sigma}$ & Moyenne & 114,1 & $\pm 37,4 b$ & 304,6 & $\pm 34,8 \mathrm{a}$ & 4,3 & $\pm 1,8$ \\
\hline$\vec{c}$ & $0 \mathrm{JAL}$ & 144,5 & $\pm 19,4 a$ & 255,7 & $\pm 57,2 \mathrm{a}$ & 3,3 & $\pm 0,6 \mathrm{~b}$ \\
\hline हू & $10 \mathrm{JAL}$ & 119,1 & $\pm \quad 14,7 a b$ & 272,4 & $\pm \quad 44,0 \mathrm{a}$ & 5,5 & $\pm 1,5 a$ \\
\hline $\begin{array}{l}E \\
\Phi\end{array}$ & $20 \mathrm{JAL}$ & 103,6 & $\pm \quad 17,6 b$ & 268,6 & $\pm 54,1 \mathrm{a}$ & 4,3 & $\pm \quad 0,9 a b$ \\
\hline อั & $30 \mathrm{JAL}$ & 85,7 & $\pm 38,3 \mathrm{bc}$ & 248,9 & $\pm 68,9 a$ & 3,5 & $\pm \quad 2,0 \mathrm{ab}$ \\
\hline$\sum_{\Sigma}^{0}$ & Sans Engrais & 63,8 & $\pm 24,1 \mathrm{c}$ & 266,2 & $\pm 57,0 \mathrm{a}$ & 2,7 & $\pm 1,2 b$ \\
\hline Effet variét & & 0,004 & & 0,000 & & 0,06 & \\
\hline Effet mome & & 0,000 & & 0,671 & & 0,00 & \\
\hline Interaction & & 0,498 & & 0,654 & & 0,52 & \\
\hline
\end{tabular}

JAL : Jour Après Levée ; PEE : Poids moyen de l'Epi Égrené ; PMG : Poids de Mille Grains

Quantification de la perte du poids de l'épi égrené : L'analyse du tableau 4 montre que l'apport retardé d'engrais de fond engendre de perte du poids de l'épi. La tendance observée est que plus le retard est grand plus le poids de l'épi diminue et plus encore la perte la perte est grande. La plus grande perte est observée sur les parcelles sans engrais dont elle est évaluée à plus de $50 \%$ du poids de l'épi lorsqu'on utilise l'apport d'engrais au moment de semis.

Tableau 4. Influence du retard d'apport d'engrais de fond sur la perte du poids de l'épi égrené

\begin{tabular}{l|c|c|c|c|c|c}
\hline \multirow{3}{*}{$\begin{array}{c}\text { Moment d'apport d'engrais de } \\
\text { Fond }\end{array}$} & \multicolumn{2}{|c|}{ LY1001-10 } & \multicolumn{2}{c|}{ UNILU } & \multicolumn{2}{c}{ Moyenne moment } \\
\cline { 2 - 7 } & $\begin{array}{c}\text { PEE } \\
(\mathrm{g})\end{array}$ & $\begin{array}{c}\text { Perte du PEE } \\
(\%)\end{array}$ & $\begin{array}{c}\text { PEE } \\
(\mathrm{g})\end{array}$ & $\begin{array}{c}\text { Perte du PEE } \\
(\%)\end{array}$ & $\begin{array}{c}\text { PEE } \\
(\mathrm{g})\end{array}$ & $\begin{array}{c}\text { Perte du PEE } \\
(\%)\end{array}$ \\
\hline 0 JAL & 128,2 & $-160,8$ & $-144,5$ & - \\
$10 \mathrm{JAL}$ & 108,7 & 15,2 & 129,4 & 19,5 & 119,1 & 17,6 \\
$20 \mathrm{JAL}$ & 103,6 & 19,1 & 103,6 & 35,5 & 103,6 & 28,3 \\
$30 \mathrm{JAL}$ & 67,4 & 47,4 & 104,0 & 35,3 & 85,7 & 40,7 \\
Sans Engrais & 55,0 & 57,1 & 72,6 & 54,8 & 63,8 & 55,8 \\
\hline
\end{tabular}

PEE : Poids de l'Epi Egrené (g) ; JAL : Jours après levée 


\section{DISCUSSION}

Comparaison végétative de deux génotypes de maïs : Le résultat de l'analyse de la variance a montré que le génotype LY 1001-10 fleuri tardivement que le génotype local amélioré (Unilu). Dans le contexte, actuel, de perturbation climatique, les génotypes à floraison précoce sont les plus recommandées. Ceci pourrait entraver l'appréciation du nouveau génotype (LY 1001-10). Bien que l'apparition des inflorescences mâles du génotype $L Y$ 1001-10 soit tardive, elle reste néanmoins inférieure à 70 jours et donc dans la gamme recommandé pour le climat de la province du Haut-Katanga (Senasem, 2008). Quant à la hauteur de la plante, le génotype Ly 1001-10 possède une hauteur inférieure à celle de Unilu. Dans le critère de choix de génotypes, Nyembo (2010) suggère la diffusion des génotypes de faible taille et ce critère faciliterait l'adoption du génotype LY 1001-10. En effet, les génotypes de grande taille sont susceptibles à la verse (Useni et al., 2012 ; Nyembo et al., 2013) et les épis des plantes sensibles à la verse tombent, pourrissent ou sont ravagés par des termites surtout dans la zone tropicale constituant ainsi une perte de récolte (llunga et al., 2016). Toutes fois, l'augmentation de la taille peut induire une grande production (Mohammadi et al., 2003) surtout en cas d'absence des vents violents. En outre les deux génotypes ont montré une similarité de taux de levée et de la hauteur à l'insertion de l'épi.

Effets du moment de fertilisation minérale (NPK) sur la croissance végétative du maïs: Le moment d'apports d'engrais à influer sur le taux de levée et la hauteur de la plante. La comparaison des moments d'apports d'engrais montre une faible taille sur les parcelles sans engrais alors que les autres traitements ont été similaires entre eux. Ceci révèle que le moment d'apport d'engrais n'a pas influencé la taille des plantes. Cependant, la différence entre le traitement sans engrais et les autres traitements est probablement due à la présence de l'azote dans l'engrais de fond apporté. En effet, l'azote, comme l'ont montré différents auteurs, est un élément important pour la vie de la plante et sert à construire toutes les parties vertes qui assurent la croissance et la vie des plantes (Lafond, 2004 ; FAO, $2005 b)$. Le résultat obtenu sont en adéquation avec ceux obtenus par d'autres chercheurs dans la même zone agro écologique et sur la même culture (Nyembo et al., 2012 ; Nyembo et al., 2013 ; Nyembo et al., 2015). En ce qui concerne le taux de levée, l'engrais épandu au moment de semis a induit une faible levée que les autres traitements. La déficience en eau serait responsable de ce comportement. En Effet, l'eau est un élément très important de la plante, elle participe à toutes les activités métaboliques. Intrinsèquement, les semences de maïs contiennent une proportion d'eau n'excédant pas 14\% (Delouche et al., 1973; Kelly, 1988) et ont besoin d'eau d'imbibition pour germer (Aya et al., 2011; Bewley, 1997). Elle permet aussi d'atténuer la chaleur libérée par la décomposition de l'engrais. Or, pour cette étude, l'épandage de l'engrais au moment de semis a coïncidé avec une période de sécheresse. Cela aurait déshydraté la graine inhibant ainsi la germination des graines semées. Toutefois, le taux de levée maximum n'a pas excédé $80 \%$. Ceci serait dû à ladite période de sécheresse a conduit certainement au durcissement du sol et formation des croutes superficielles empêchant ainsi la levée d'une grande partie des graines germées (Souty et Rode, 1994). Dans un contexte de faible levée, Nyembo et al. (2014) recommande le regarnissage de vide. En outre, le résultat montre que le moment d'apport d'engrais n'a pas influencé le jour à la floraison et la hauteur à l'insertion de l'épi. Ce résultat confirme l'hypothèse émise par Nyembo et al. (2013) montrant que ces deux paramètres sont plus liés au génotype qu'à l'environnement.

Rendement de deux génotypes de maïs: La comparaison de rendement de deux génotypes montre des différences non significatives. Ceci révèle que dans les conditions de la province du Haut-Katanga, le génotype LY 1001-10 pourrait substituer le génotype Unilu. Cependant, le rendement est un paramètre dépendant des autres facteurs. II est déduit à partir $\mathrm{du}$ taux de levée et du poids de l'épi égrené. L'analyse de ce dernier montre que le génotype LY 1001-10 possède des épis de petite taille et moins gros. Ceci pourrait entraver son acceptation auprès des producteurs. En outre, le génotype LY 1001-10 possède des petites graines. En effet, dans le choix de semence à utiliser, il est préférable d'avoir un génotype à petite graine. Plus les graines sont grosses, plus la quantité de semence à utiliser est grande (llunga et al., 2016). Cette faible grosseur des graines de LY 1001-10 est un avantage pour les petits producteurs. Effets du moment de fertilisation minérale (NPK) sur la productivité réelle et la perte du rendement potentielle du maïs : Les épis trouvés sur les parcelles non fertilisées avaient un poids faible par rapport à ceux des parcelles fertilisées. Ceci serait dû à la disponibilisation des éléments nutritifs comme l'azote par l'engrais car permettant d'accroitre le rendement et ses composantes (Samira et al., 1998 ; Torbert et al., 2001). Plusieurs auteurs recherches ont trouvé des résultats similaires montrant ainsi, l'importance de l'engrais dans l'accroissement du poids de l'épi (Asghar et al., 2010 ; 
Jaliya et al., 2008; Nyembo et al., 2012). Bien que l'apport de NPK ait accru le poids de l'épi, cependant le moment d'apport est d'importance capitale. En effet, épandre l'engrais au moment de semis permet d'obtenir des épis de grand poids que lorsqu'il est épandu 10, 20 ou 30 jours après levée. Cette situation serait due à la synchronisation entre la libération des éléments nutritifs par l'engrais et leur assimilation par la plante étant donné que la décomposition du NPK est lente. Ainsi, son application à 0 et 10 jours après levée a permis à la plante d'utiliser les éléments libérés quand elle en avait besoin contrairement aux parcelles fertilisées 20 et 30 jours après levée. De plus, au stade juvénile, la demande est élément nutritif tel que l'azote est faible et qu'elle augmente lorsqu'on s'approche de la floraison. Ceci combiné à sa lente décomposition aurait induit une diminution du poids de l'épi au fur et à mesure l'apport de l'engrais avoisinait la floraison. L'un des objectifs de cette étude était de déterminer la période d'épandage de l'engrais ternaires qui accroit le mieux le rendement de la culture. En scrutant les résultats, le meilleur rendement a été obtenu en apportant le NPK 10 jours après levée contrairement à l'épandage au moment de semis. Bien ce dernier ait donné des gros épis, son faible taux de levée $(<50 \%)$ a induit une diminution du rendement. Ceci confirme les allégations de Useni et al. (2014) montrant que l'augmentation du nombre d'épi entraine un accroissement du rendement étant donné que le rendement est la somme de poids individuels de chaque épi. En outre, la comparaison de taux de levée a montré que toutes les parcelles n'ayant pas reçues l'engrais au moment de semis avaient un taux de levée similaire. Cependant, le rendement de celles fertilisées 10 jours

\section{CONCLUSION}

L'objectif de la présente étude était d'évaluer le comportement du maïs (génotype Unilu et LY 1001-10) sous l'effet de différents moments d'apport d'engrais tertiaire. Les résultats obtenus ont montré que l'application d'engrais de fond (NPK) permet d'obtenir des plantes de grandes tailles ayant un poids de l'épi égrené ainsi que le rendement élevé. Cependant, l'application du NPK au moment de semis induit un faible taux de levée, un grand poids de l'épi mais similaire NPK appliqué dix jours après levée. Toutefois, le poids de l'épi diminue au fur et à mesure que l'application de l'engrais avoisine le

\section{RÉFÉRENCES}

Akbar H., Miftahullah M.T., Jan A., Ihsanullah A., 2002. Yield potential of sweet corn as influenced by après levée a été le plus élevé suit au poids élevé des épis obtenus contredisant ainsi les hypothèses de l'augmentation du rendement basée uniquement sur l'augmentation de la densité prônée par Emam (2001) ; Akbar et al. (2002) et Mojtaba et al. (2012). Ainsi, le taux de levée doit être considéré au même degré que la densité et le poids moyen de l'épi égrené comme facteur directement proportionnel à l'augmentation du rendement car à une même densité, plus le taux de mortalité est grand plus faible sera le rendement. En évaluant les pertes du poids de l'épi qui traduit le rendement potentiel dû au retard d'apport d'engrais. Le résultat montre une tendance régressive du poids de l'épi lors que l'apport s'approche de la floraison. La perte a été plus faible à 10 jours (17\%) qu'à 30 jours (40\%). Ce dernier taux montre l'inefficacité de l'engrais NPK lorsqu'il est apporté au 30 ème jour après levée. Par ailleurs, faire une culture sans engrais dans le sol ferralitique pourrait réduire d'au moins $55 \%$ votre production. Toutefois, l'apport de l'engrais 30 jours après semis a accru le rendement potentiel ou le poids de l'épi égrené de $15 \%$ que le traitement sans engrais (Tableau 4). Cette augmentation parait trop faible au regard des coûts engendre par l'achat et l'épandage du NPK $\left(300 \$^{*} h a^{-1}\right)$ dans la province du Haut-Katanga. Les observations faites montrent que l'épandage de l'engrais au moment de semis est une technique à encourager lorsqu'il ne pas suivi d'une période de sécheresse. Dans le cas contraire, apporter l'engrais 10 jours après levée permet d'obtenir des épis ayant un poids plus proche d'un épandage d'engrais au moment de semis. Toutefois, l'application de l'engrais devient inefficace au fur et à mesure que le décalage entre le semis et moment d'application est grand.

jour à la floraison et la perte devient de plus en plus grande. La perte du poids de l'épi égrené et du rendement potentiel est plus élevée, dans la province du Haut-Katanga, lorsque le maïs est cultivé sans engrais et qu'il est préférable de cultivé sans engrais que d'apporter l'engrais vers le 30 ème jour après levée. L'application du NPK au moment de semis est très avantageuse si elle n'est pas suivie d'une période sèche. Dans le cas contraire, apporter le NPK 10 jours après levée est une technique à encourager et que l'allongement du décalage d'application est néfaste pour la production.

different levels of nitrogen and plant population. Asian J. Plant Sci. 1: 631-633 
Amede T., 2003. Opportunities and Challenges in Reversing Land degradation: The Regional Experience. In : Amede, T(ed), Natural resource degradation and environmental concerns in the Amhara National Regional State: Impact on Food Security. Ethiopian Soils Sci. Soc, p. 173183

Asghar A., Ali A., Syed W. H., Asif M., Khaliq T., Abid A. A., 2010. Growth and yield of maize (Zea mays L.) cultivars affected by NPK application in different proportion. Pakistan journal of Science 62(4): 211-216

Aya A., Irié V., Kouamé L., Irié A., 2011. Bases génétiques et biochimiques de la capacité germinative des graines : implications pour les systèmes semenciers et la production alimentaire. Sciences \& Nature Vol. $8 N^{\circ} 1: 119$ $-137$

Bewley J., 1997. Seed Germination and Dormancy. The Plant Cell. 9(7): 1055-1066

Bot A., Benites J., 2005. The importance of soil organic matter: key to drought-resistant soil and sustained food production (No. 80). Food \& Agriculture Org. $95 \mathrm{p}$

Cunha T.J.F., Madari B.E., Canellas L.P, Ribeiro L.P., de MeloBenites V., de Araujo S. G., 2009. Soil organic matter and fertility of the anthropogenic dark earths (Terra Preta de Indio) in the Brazilian Amazon basin, RevistasBrasileira de Ciencia do Solo 33 : 85-93

Delouche J. C., Matthes R. K., Dougherty G. M., Boyd A. H., 1973. Storage of seed in sub-tropical and tropical regions. Seed Sci. \&Technol1 : 671-700

Dembélé N. N., 2001. Sécurité alimentaire en Afrique Sub-saharienne: Quelle Stratégie de Réalisation? Rapport d'évaluation Michigan State University/PASIDMA/Chambre d' Agriculture du Mali, Bamako. 25p

Emam Y., 2001. Sensitivity of grain yield components to plant population density in non-prolific maize (Zea mays) hybrids. Indian J. Agric. Sci. 71(6):367-370

Ezeh Alex C., Bongaarts J., Mberu B., 2012. Global population trends and policy options. The Lancet $380.9837: 142-148$

FAO, 2005a. New_LocClim : Local Climate Estimator. FAO Environment and Natural Resources Working Paper, $\mathrm{N}^{\circ} 20$

FAO, 2005b. Notions de nutrition des plantes et de fertilisation des sols. Manuel de formation, Projet Promotion de l'Utilisation des Intrants agricoles par les Organisations de Producteurs du Niger, Rome, $24 \mathrm{p}$

Giroux M., N'DayegamiyeA., Royer R., 2007. Effet des apports d'automne et printemps de fumiers et des boues mixtes de papetières sur le rendement, la qualité de la pomme terre et efficacité de l'azote. Agrosolutions, 18(1): 25-34

Henao J., Baanante C., 2006. Agricultural production and soil nutrient mining in Africa: Implication for resource conservation and policy development. IFDC Tech. Bull. International Fertilizer Development Center. Muscle Shoals, Al. USA, $13 p$

Ilunga T. H., Muganguzi N. T., Kidinda K. L., Banza M. J., Nsenga N. S., Mpoyo M. G., Tshipama T. D., Lukusa M. L. and Nyembo K. L., 2015. Evaluation of Maize Response (Zea mays L.) to Various Modes and Moments of Chicken Manure Spreading in Lubumbashi, DR Congo. American Journal of Plant Nutrition and Fertilization Technology 5 (3): 96-105

llunga T.H., Sadiki M.P., Tshibuyi K.B., Nsenga N. S., Mazinga K. M., Nyembo K. L. \&Baboy L. L.. 2016. Les faibles doses de biomasses vertes de légumineuses, des bons substituts des engrais minéraux dans la maïsiculture à Lubumbashi ? Afrique Science.12(2) : 317-325

Jaliya M. M., Falaki A. M., Mahmud M., Sani Y. A., 2008. Effects of sowing date and NPK fertilizer rate on yield and yield components of quality protein maize (Zea mays L.). ARPN Journal of Agricultural and Biological Science, 3(2): 23-29

Kasongo L.M.E., Mwamba M.T., Tshipoya M.P., Mukalay M.J., Useni S.Y., Mazinga K.M., Nyembo K.L., 2013. Réponse de la culture de soja (Glycine max L. (Merril) à l'apport des biomasses vertes de Tithonia diversifolia (Hemsley) A. Gray comme fumure organique sur un Ferralsol à Lubumbashi, R.D. Congo. Journal of Applied Biosciences63 : 4727-4735

Kelly A.F., 1988. Principles of Seed Growing. Longman Group Ltd., UK. 36-55

LafondJ., 2004. Fractionnement de la fertilisation azotée minérale et organique : Effet sur la productivité du canola de printemps et sur les nitrates du sol. Can. J. Soil Sci. 84: 491-501

Lutz W., Sanderson W., Scherbov S., 2001. The end of world population growth. Nature, 412 (6846); 543-545

Mohammadi SA, Prasanna BM, Singh NN., 2003. Sequential path model for determining 
interrelationship among grain yield and related characters in maize. Crop Sci. 43: 1690-1697

Mojtaba N.K.K., Saied K.K., Taheri G., 2012. Effects of plant density and variety on some of morphological traits, yield and yield components of baby corn (Zea mays L.). International Research Journal of Applied and Basic Sciences $3(10): 2009-2014$

Nyembo K.L, 2010. Augmentation du rendement du maïs par l'exploitation de l'effet hétérosis des hybrides produits au Katanga, République Démocratique du Congo. Thèse de doctorat, Faculté des sciences agronomiques, Université de Lubumbashi, 157p.

Nyembo K.L., Useni S.Y., Mpundu M.M., Bugeme M.D., Kasongo L.E., Baboy L.L., 2012. Effets des apports des doses variées de fertilisants inorganiques (NPKS et Urée) sur le rendement et la rentabilité économique de nouvelles variétés de Zea mays L. à Lubumbashi, Sud Est de la RD Congo. Journal of Applied Biosciences 59: 4286-4296

Nyembo K.L., Useni S.Y., Chukiyabo K.M., Tshomba K.J., Ntumba N.F., Muyambo M.E., Kapalanga K.P., Mpundu M.M., Bugeme M.D., Baboy L.L., 2013. Rentabilité économique du fractionnement des engrais azotés en culture de mais (Zea mays $L$.) : cas de la ville de Lubumbashi, sud-est de la RD Congo. Journal of Applied Biosciences 65:4945-4956

Nyembo K.L., Useni S.Y., Mpundu M.M., Kyungu K., Baboy L.L., 2014. Evaluation des nouvelles variétés de maïs (Zea mays $L$.) en provenance de la firme Pannar dans les conditions climatiques de la région de Lubumbashi (RD Congo). E-Revue unilu. 1: 52-61

Nyembo K. L., Banza M. J., Salima B. S. N., Tshipama T. D., Kiluba K. M., Mpoyo M. G., Muteba K. M., 2015. Les faibles doses d'engrais azotés ne permettront pas d'optimiser le rendement des nouvelles variétés de maïs dans la région de Lubumbashi (RD Congo). International Journal of Innovation and Applied Studies 12: 176-182

Pang X.P., Letey J., 2000. Organic farming : Challenge of timing nitrogen availability to crop nitrogen requirements. Soil Sci. Soc. Amer. J. 64: 247253

Qafoku N.P., Van Ranst E., Noble A., Baert G., 2004. Variable charge soils: their mineralogy, chemistry and management. Adv. Agron. 84: $159-215$
Samira M., Hussein A., Haikeland M.A. and El-Masry A., 1998. Effect of some preceding crops, hill spacing and nitrogen fertilization on yield attributes and grain yield of maize under reclaimed sandy soil conditions in East Delta. In Proc. 8th Conf. Agron., Suez Canal Univ., Ismailia, Egypt, 28-29

Senasem., 2008. Politique nationale du développement du sous-secteur de semences. Appui au projet ASS, Minagri, Kinshasa, $56 \mathrm{p}$.

Souty N., Rode N., 1994. La levée des plantules au champ : un problème mécanique ? Sécheresse5 : 13-22

Torbert H.A., Potter K.N., Morrison J.E., 2001. Tillage system, fertilizer nitrogen rate and timing effect on corn yields in the Texas Blackland prairie.Agronomy Journal 93: 1119-1124

Useni S.Y., Baboy L.L., Nyembo K.L., Mpundu M.M., 2012. Effets des apports combinés de biodéchets et de fertilisants inorganiques sur le rendement de trois variétés de Zea mays $L$. cultivées dans la région de Lubumbashi. Journal of Applied Biosciences 54 : 3935-3943

Useni S.Y., Chukiyabo K.M., Tshomba K.J., Muyambo M.E., Kapalanga K.P., Ntumba N.F., Kasangij K.P., Kyungu K.A., Baboy L.L., Nyembo K.L. \&Mpundu M.M., 2013. Utilisation des déchets humains recyclés pour l'augmentation de la production du maïs (Zea mays L.) sur un ferralsol du Sud-Est de la RD Congo. Journal of Applied Biosciences 66:5070-5081

Useni S. Y., Kanyenga L. A., Assani B.L., Ekondo O. A., Baboy L. L., Ntumba K. B., Mpundu M. M., Nyembo K. L., 2014.Influence de la date de semis et de la fertilisation inorganique sur le rendement de nouveaux hybrides de maïs (Zea mays L.) à Lubumbashi. Journal of Applied Biosciences 76:6316-6325 